\title{
Correction to: Influence of Tibetan Plateau on the North American summer monsoon precipitation
}

\author{
Qin Wen ${ }^{1,2,8,9} \cdot$ Zixuan $\mathrm{Han}^{3} \cdot$ Haijun Yang ${ }^{4} \cdot$ Jianbo Cheng ${ }^{5} \cdot$ Zhengyu Liu $^{6} \cdot$ Jian Liu $^{1,2,7}$
}

Published online: 22 July 2021

(c) Springer-Verlag GmbH Germany, part of Springer Nature 2021

Correction to: Climate Dynamics

https://doi.org/10.1007/s00382-021-05857-y

In the original publication of the article, the order of affiliation was incorrect. The correct information is as follows,

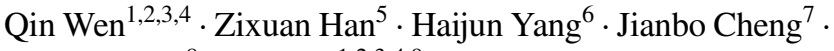
Zhengyu Liu ${ }^{8} \cdot \operatorname{Jian} \mathrm{Liu}^{1,2,3,4,9}$

${ }^{1}$ School of Geography, Nanjing Normal University, Nanjing 210023, China

${ }^{2}$ Jiangsu Center for Collaborative Innovation in Geographical Information Resource Development and Application, Nanjing 210023, China

${ }^{3}$ Key Laboratory of Virtual Geographic Environment (Nanjing Normal University), Ministry of Education, Nanjing 210023, China

${ }^{4}$ Open Studio for Ocean-Climate-Isotope Modeling, Pilot National Laboratory for Marine Science and Technology (Qingdao), Qingdao 266100, China

The original article can be found online at https://doi.org/10.1007/ s00382-021-05857-y.

Qin Wen

wenq@pku.edu.cn

1 School of Geography, Nanjing Normal University, Nanjing 210023, China

2 Jiangsu Center for Collaborative Innovation in Geographical Information Resource Development and Application, Nanjing 210023, China

3 Key Laboratory of Virtual Geographic Environment (Nanjing Normal University), Ministry of Education, Nanjing 210023, China

4 Open Studio for Ocean-Climate-Isotope Modeling, Pilot National Laboratory for Marine Science and Technology (Qingdao), Qingdao 266100, China
${ }^{5}$ College of Oceanography, Hohai University, Nanjing 210024, China

${ }^{6}$ Department of Atmospheric and Oceanic Sciences, Institute of Atmospheric Science and CMA-FDU Joint Laboratory of Marine Meteorology, Fudan University, Shanghai 200438, China

${ }^{7}$ School of Environmental Science and Engineering, Yancheng Institute of Technology, Yancheng 224051, China

${ }^{8}$ Department of Geography, Ohio State University, Columbus, OH, USA

${ }^{9}$ Jiangsu Provincial Key Laboratory for Numerical Simulation of Large Scale Complex Systems, School of Mathematical Science, Nanjing Normal University, Nanjing 210023, China

The original article has been corrected.

Publisher's Note Springer Nature remains neutral with regard to jurisdictional claims in published maps and institutional affiliations.

5 College of Oceanography, Hohai University, Nanjing 210024, China

6 Department of Atmospheric and Oceanic Sciences, Institute of Atmospheric Science and CMA-FDU Joint Laboratory of Marine Meteorology, Fudan University, Shanghai 200438, China

7 School of Environmental Science and Engineering, Yancheng Institute of Technology, Yancheng 224051, China

8 Department of Geography, Ohio State University, Columbus, OH, USA

9 Jiangsu Provincial Key Laboratory for Numerical Simulation of Large Scale Complex Systems, School of Mathematical Science, Nanjing Normal University, Nanjing 210023, China 